\title{
Flocculation Efficiency and Mechanism of Carbamazepine by Microbial Flocculant Extracted from Klebsiella pneumoniae J1
}

\author{
Jie Xing $\mathbb{C}^{1,2,3}$ Nanzhe Song, ${ }^{2}$ Xiangwei Chen, ${ }^{1}$ Ang $\mathrm{Li}^{4},{ }^{4}$ and Hongwei $\mathrm{Ni} \mathbb{D}^{3}$ \\ ${ }^{1}$ Northeast Forestry University, Harbin 150040, China \\ ${ }^{2}$ Heilongjiang Provincial Research Academy of Environmental Sciences, Harbin 150056, China \\ ${ }^{3}$ Institute of Natural Resources and Ecology, HAS, Harbin 150040, China \\ ${ }^{4}$ State Key Laboratory of Urban Water Resource and Environment, School of Environment, Harbin Institute of Technology, \\ Harbin 150090, China \\ Correspondence should be addressed to Jie Xing; 18686885851@163.com and Hongwei Ni; nihongwei2000@163.com
}

Received 25 August 2020; Revised 18 September 2020; Accepted 13 October 2020; Published 18 November 2020

Academic Editor: Jin Li

Copyright (C) 2020 Jie Xing et al. This is an open access article distributed under the Creative Commons Attribution License, which permits unrestricted use, distribution, and reproduction in any medium, provided the original work is properly cited.

\begin{abstract}
The microbial flocculant (MFX) extracted from Klebsiella pneumoniae J1 was used to remove carbamazepine in prepared wastewater and domestic sewage. The influence factors and flocculation mechanism were studied. The optimal carbamazepine removal conditions for MFX were $\mathrm{pH}$ of $7-8,7 \mathrm{~mL}$ of flocculant, $0.1 \mathrm{~mL}$ of coagulant, and $35^{\circ} \mathrm{C}$, and the removal rate reached 81.75\%. MFX was efficient in the removal of carbamazepine in both domestic sewage $(75.03 \%)$ and secondary sedimentation tank effluent (69.76\%). The pseudo-first-order kinetic equation fitted the adsorption process better than the pseudo-secondorder kinetic equation, which suggested that the adsorption was not pure chemical adsorption. The analysis of floc size suggested that the repulsive force between carbamazepine and MFX was weakened under alkalescent conditions, which can help the growth and coherence of flocs and increase the carbamazepine removal efficiency. Enough dosage of MFX can generate larger flocs, but excessive dosage of MFX will decrease the carbamazepine removal rate because of increase in electrostatic repulsion. The analysis of 3D-EEM and FTIR suggested that hydroxyl, amino, and carboxyl in MFX played an important role in the removal of carbamazepine. As an eco-friendly and highly efficient microbial flocculant, MFX has potential for practical applications in carbamazepine removal.
\end{abstract}

\section{Introduction}

Carbamazepine (CBZ), a typical pharmaceutical and personal care product (PPCP) [1-3], is a medicine for the treatment of epilepsy, depression, glossopharyngeal neuralgia, trigeminal neuralgia, and central partial diabetes insipidus [4]. Toxicological experiments show that the LD50 of carbamazepine is $4205 \mathrm{mg} / \mathrm{kg}$ (for rats). Carbamazepine has been detected in sewage treatment stations since 1998, and it has been found that the concentration of carbamazepine in water is higher than that of other PPCPs in recent years [5]. Carbamazepine widely exists in the effluent of urban sewage plants, surface water, and soil. Carbamazepine may eventually enter drinking water and groundwater through environmental migration and transformation $[3,6]$.
It can be seen from the pollution status of carbamazepine in the environment that carbamazepine widely exists in various environmental media after migration and transformation, and it has good stability and durability, allowing it to exist in the environment for a long time $[7,8]$. Therefore, it is urgent to improve the removal efficiency of carbamazepine in the research of PPCP pollutants.

Microbial flocculants (MFX), extracted from microorganisms, widely exist and are nontoxic and eco-friendly [9-11]. There are numerous chemical groups (e.g. hydroxyl, carboxyl, and amino) in MFX, which allow MFX to have good combining capacity with pollutants and have good flocculation efficiency [12-15]. In addition to the removal of turbidity of wastewater, it has been proved that MFX can treat wastewaters that contain heavy metals [16-18] and antibiotics [19-21]. 
Because there is no secondary pollution of MFX, it is one of the most promising materials to treat wastewater [22].

Until now, most studies on MFX used prepared solution to study the removal efficiency of MFX. Actually, the constituents in actual wastewater are much more complex than those in a prepared solution [23]. There are many other contaminants in actual wastewater, and these contaminants could compete against carbamazepine, which will decrease the carbamazepine removal efficiency by MFX. Therefore, besides prepared solutions, it is also important to conduct experiments on carbamazepine removal in actual wastewater.

In this study, MFX was used to remove carbamazepine from wastewater. The carbamazepine removal efficiency was tested under different conditions ( $\mathrm{pH}$, dosage of MFX, dosage of coagulant, and temperature). Considering the practical application of MFX, the carbamazepine removal efficiency by MFX was also studied in domestic sewage and in secondary sedimentation tank effluent. The adsorption kinetics and the mechanism of carbamazepine flocculation were studied.

\section{Materials and Methods}

2.1. Materials and Reagents. The extraction of MFX from Klebsiella pneumoniae J1 (CGMCC No. 6243) was described in our previous work [19]. Briefly, J1 was inoculated and fermented to produce MFX. MFX was extracted by alcohol extraction and was purified by dialysis in ultrapure water. Dried MFX powder was obtained by vacuum freeze drying and stored at $4^{\circ} \mathrm{C}$.

Carbamazepine, $\mathrm{CaCl}_{2}$, and the reagents used to prepare MFX were purchased from Bailingwei Technology Co., Ltd. Ultrapure water $(18 \mathrm{M} \Omega / \mathrm{cm}$, Milli-Q), and analytical-grade reagents were used in this work.

2.2. Batch Adsorption Experiments. The stock carbamazepine solution $(5 \mathrm{mg} / \mathrm{L})$ was prepared by dissolving carbamazepine in ultrapure water and stored at $4^{\circ} \mathrm{C}$ for later use. The stock carbamazepine solutions were diluted appropriately to obtain the experimental solutions. MFX (1 g/L) and coagulant aid $\left(\mathrm{CaCl}_{2}, 10 \%\right)$ were added to the initial carbamazepine solution $(1 \mathrm{mg} / \mathrm{L}, 1 \mathrm{~L})$. After flocculating and stirring for a period of time, the samples were allowed to stand for $20 \mathrm{~min}$. After adsorption, MFX was separated by $0.45 \mu \mathrm{m}$ filter membrane and the concentration of carbamazepine was determined by high-performance liquid chromatography (HPLC, Shimadzu, LC-10A (SPD/RID/RFD), Japan). All experimental data were the average of triplicate measurements. The adsorption capacity $\left(q_{e}, \mathrm{mg} / \mathrm{g}\right)$ of carbamazepine by MFX was calculated as follows:

$$
q_{e}=\left(C_{0}-C_{e}\right) \frac{V}{M}
$$

where $C_{0}$ and $C_{e}$ are the initial and equilibrium carbamazepine concentrations $(\mathrm{mg} / \mathrm{L})$, respectively, $V$ is the volume of the carbamazepine operating solution (L), and $M$ is the dose of $\operatorname{MFX}(\mathrm{g})$.
MFX was also used to adsorb carbamazepine in domestic sewage and sewage from the secondary settling tank from the Harbin Taiping Sewage Treatment Plant. The adsorption process was similar to that of carbamazepine solution. The difference was that the concentration of carbamazepine in the domestic sewage and the sewage from the secondary settling tank was much lower than the prepared carbamazepine solution; therefore, the samples were pretreated via ethyl acetate liquid-liquid extraction. To be specific, the same volume of ethyl acetate was added to the filtered supernatant, the sample was shaken for $10 \mathrm{~min}$, and then, it was allowed to stand for layering. The supernatant liquid was extracted, shaken for $5 \mathrm{~min}$, and then allowed to stand for layering. The supernatant liquid was extracted. After rotary evaporation and nitrogen blowing of the collected upper liquid, it was redissolved with chromatographic grade methanol. The concentration of carbamazepine was measured by highperformance liquid chromatography (HPLC, Waters, USA). Three parallel samples were prepared for each sample. Carbamazepine $(20 \mu \mathrm{g} / \mathrm{L})$ was used as a standard sample to measure the recovery rate of the spiked standard.

2.3. Adsorption Kinetics. The adsorption kinetics of MFX for carbamazepine were discussed using the pseudo-first-order and pseudo-second-order kinetic models. The initial concentration of carbamazepine was at a $\mathrm{pH}$ of 7 , with $\mathrm{CaCl}_{2}$ $(0.1 \mathrm{~mL}, 10 \%)$ as a coagulant aid. The adsorption times were $5,10,15,20,30,40,50$, and $60 \mathrm{~min}$, respectively. The pseudofirst-order and pseudo-second-order kinetic models are displayed in

$$
\begin{gathered}
\log \left(q_{e}-q_{t}\right)=\log \left(q_{e}\right)-\frac{k_{1}}{2.303} t \\
\frac{t}{q_{t}}=\frac{1}{k_{2} q_{e}^{2}}+\frac{t}{q_{e}},
\end{gathered}
$$

where $q_{e}$ and $q_{t}$ are the equilibrium adsorption capacity and adsorption capacity at a certain moment, respectively $(\mathrm{mg} / \mathrm{g})$, $t$ is the adsorption time ( $\mathrm{min}$ ), and $k_{1}$ and $k_{2}$ are the rate constants of pseudo-first-order and pseudo-second-order kinetic models, respectively.

\subsection{Analysis of Mechanisms of Carbamazepine Adsorption on} MFX. A granulometer (Malvern MS2000, UK) was used to monitor the growth of different microbial flocculants and to calculate the growth rate of the flocs in the adsorption process. The functional groups were determined by Fourier transform infrared spectroscopy (FTIR, Perkin-Elmer spectrum 100). Three-dimensional fluorescence spectrophotometry (3D-EEM, FP6500, JASCO, Japan) was used to obtain EEM spectra, and the fluorescence response was obtained by scanning the emission spectra from 220 to $650 \mathrm{~nm}$ and by varying the excitation wavelength from 220 to $500 \mathrm{~nm}$. Fluorescence quenching, completed by adding carbamazepine into the MFX solution, was used to assess the adsorption characteristics. 


\section{Results and Discussion}

3.1. Factors That Influence the Removal Efficiency of Carbamazepine via MFX. Generally, to evaluate the adsorption capacity of MFX, the removal efficiency of a pollutant needs to be tested under different conditions. In this study, the adsorption performance of carbamazepine by MFX was studied under different conditions by changing the $\mathrm{pH}$, dosage of MFX, dosage of coagulant aid, and temperature, respectively.

3.1.1. $p H$. The $\mathrm{pH}$ value is an important parameter in the adsorption process. The $\mathrm{pH}$ not only affects the functional groups of MFX but also affects the surface charge of carbamazepine [24, 25]. The removal rate of carbamazepine by MFX was tested at $\mathrm{pH}=5-10$, and the results are shown in Figure 1(a). It was obvious that the removal rate of carbamazepine by MFX increased from $\mathrm{pH}=5$ to $\mathrm{pH}=7$ and decreased from $\mathrm{pH}=7$ to $\mathrm{pH}=10$. MFX showed a poor carbamazepine removal effect under acidic conditions. At $\mathrm{pH}$ $=5$, the removal rate was only $5.14 \%$. However, the carbamazepine removal rate became better under nonacidic conditions. At $\mathrm{pH}=7, \mathrm{MFX}$ had the best carbamazepine removal rate $(65.26 \%)$, and the removal rate decreased apparently at $\mathrm{pH}=9-10$. This suggests that both acidic and overly alkaline conditions are not suitable for the removal of carbamazepine by MFX. This was because the change of $\mathrm{pH}$ conditions changed the quantity and properties of surface charges of both MFX and carbamazepine, and overly acidic and overly alkaline conditions can weaken the neutralization effect and hinder the aggregation reaction between MFX and carbamazepine [20]. Therefore, the optimal $\mathrm{pH}$ range for the removal of carbamazepine by MFX is $7-8$.

3.1.2. Dosage of MFX. The dosage of MFX is another important factor that can affect the removal rate of carbamazepine [26]. The removal rate of carbamazepine by MFX was tested after adding a different dosage of MFX, and the results are shown in Figure 1(b). The carbamazepine removal rate increased when the dosage increased from $1 \mathrm{~mL}$ to $7 \mathrm{~mL}$ but then decreased with the increase of dosage of MFX. MFX performed the best when its dosage was $7 \mathrm{~mL}$ and the maximum removal rate was $73.06 \%$. When $9 \mathrm{~mL}$ dosage of MFX was added to carbamazepine, the removal rate showed a downward trend. This was because when the dosage of MFX was low, the quantity of MFX was not enough to remove carbamazepine even if MFX reached adsorption saturation. However, when the dosage was too high, the excessive MFX could influence the charge property of the entire system and destroy the balance of charge, which subsequently decreased the carbamazepine removal rate. This result was similar to those in other studies [27].

3.1.3. Dosage of Coagulant Aid. Coagulant aids can improve the flocculation conditions by adjusting the charge property of the system [21]. In this study, $\mathrm{CaCl}_{2}$ was added as a coagulant aid, and the carbamazepine removal rate of MFX with different dosage of coagulant aid is shown in Figure 1(c). The $\mathrm{CaCl}_{2}$ dosages of samples 1 to 6 were $0 \mathrm{~mL}, 0.1 \mathrm{~mL}$, $0.3 \mathrm{~mL}, 0.5 \mathrm{~mL}, 1 \mathrm{~mL}$, and $1.5 \mathrm{~mL}$, respectively. It can be seen that the carbamazepine removal rate by MFX was 54.73\% when no coagulant was added, which proves that MFX can remove carbamazepine alone. When the dosage was $0.1 \mathrm{~mL}$, the carbamazepine removal rate increased and reached the maximum removal rate of $77.17 \%$. However, with the increase of $\mathrm{CaCl}_{2}$ dosage, the carbamazepine removal rate decreased gradually. This was because carbamazepine was removed by MFX through adsorption bridging between macromolecules, and the coagulant aid can strengthen the adsorption bridging by adjusting the charge property of the system. However, excessive dosage of the coagulant aid will introduce excessive positive charges, which bind to the negatively charged adsorption sites in MFX, hindering the connection of carbamazepine and MFX. Therefore, the optimal dosage of coagulant aid needs to be determined by adsorption experiments. In this study, the optimal dosage was $0.1 \mathrm{~mL}$.

3.1.4. Temperature. Temperature is another important factor that can affect the carbamazepine removal efficiency by MFX [26]. Figure 1(d) shows the carbamazepine removal rate by MFX at $15^{\circ} \mathrm{C}, 20^{\circ} \mathrm{C}, 25^{\circ} \mathrm{C}, 30^{\circ} \mathrm{C}$, and $35^{\circ} \mathrm{C}$, respectively. It can be seen that the removal rate increased as the temperature increased. The removal rate was only $22.06 \%$ at $15^{\circ} \mathrm{C}$, but increased to $81.75 \%$ at $35^{\circ} \mathrm{C}$. Generally, an appropriate temperature increase can accelerate the random movement between molecules, which then increase the collision probability among molecules and increase the carbamazepine removal rate by MFX. However, too high of a temperature will lead to the inactivation of MFX, which will decrease the carbamazepine removal rate [28]. In this study, MFX performed the best at $35^{\circ} \mathrm{C}$, suggesting MFX was effective even at $35^{\circ} \mathrm{C}$.

In summary, the optimal carbamazepine removal conditions for MFX were $\mathrm{pH} 7-8,7 \mathrm{~mL}$ of flocculant, $0.1 \mathrm{~mL}$ of coagulant, and $35^{\circ} \mathrm{C}$. The removal rate reached $81.75 \%$.

3.2. Carbamazepine Removal from Domestic Sewage. The above experiments prove that MFX has a good carbamazepine removal efficiency for a prepared carbamazepine solution $(1 \mathrm{mg} / \mathrm{L})$. However, the carbamazepine concentration in domestic sewage is usually tens to hundreds of $\mu \mathrm{g} / \mathrm{L}$. Moreover, the constituents in domestic sewage are much more complex than a prepared carbamazepine solution [23]. Although domestic sewage contains a large number of suspended solid colloidal particles that are conducive to the formation of flocs, the competition for the adsorption sites on MFX between carbamazepine and other constituents can lead to the decrease of carbamazepine removal rate. Figures 2(a) and 2(b) show the removal rate in domestic sewage and in secondary sedimentation tank effluent. It can be seen that MFX was efficient in the removal of carbamazepine both in domestic sewage $(75.03 \%)$ and in secondary sedimentation tank effluent (69.76\%), which suggested that MFX had a good removal effect on carbamazepine.

3.3. Adsorption Kinetics. Adsorption kinetics is important when studying adsorption mechanism. The kinetics analyzes the effects of adsorption time on the adsorption of pollutants 


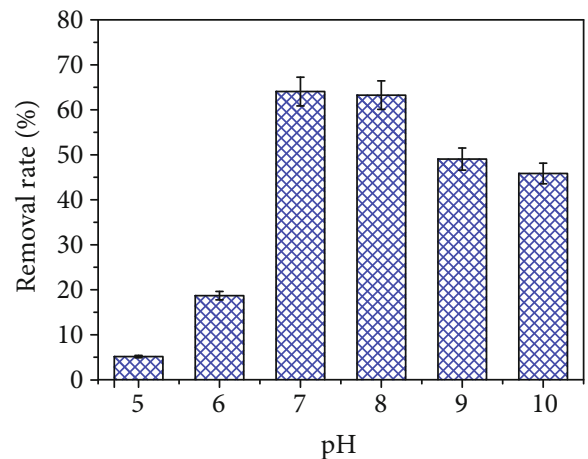

(a)

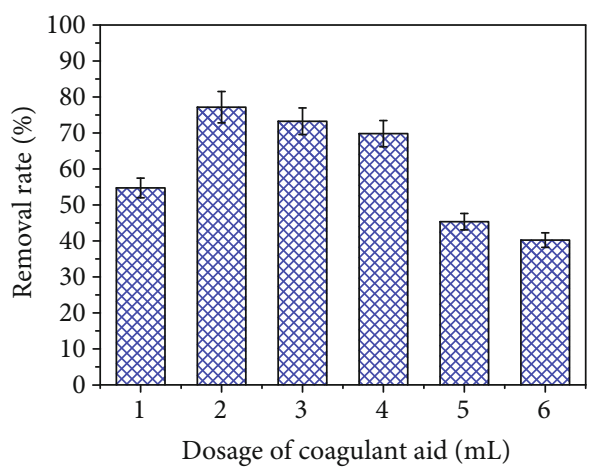

(c)

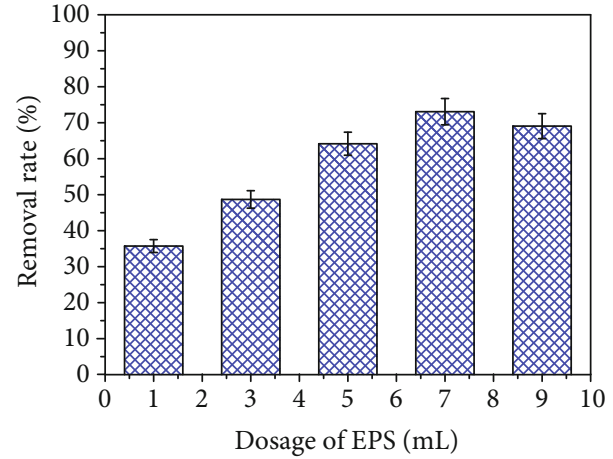

(b)

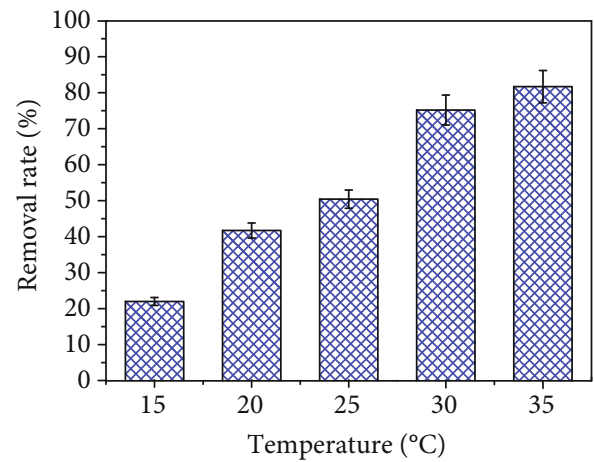

(d)

Figure 1: Removal efficiency of carbamazepine on MFX at different conditions: (a) pH, (b) dosage of MFX, (c) dosage of coagulant aid, and (d) temperature.

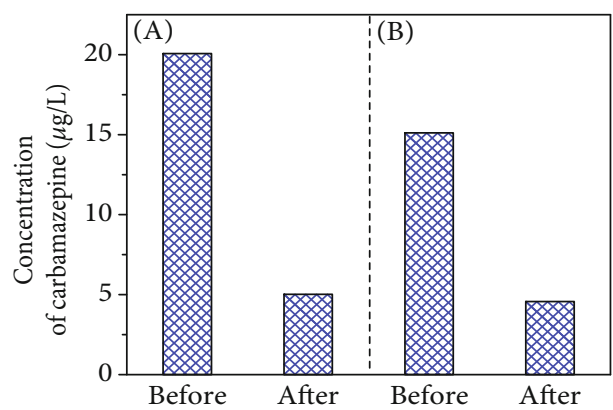

FIgURE 2: Removal efficiency of carbamazepine on MFX: (a) domestic sewage and (b) secondary sedimentation tank effluent.

by describing the adsorption rate and then explores the adsorption mechanism. Usually, the first-order, secondorder, pseudo-first-order, and pseudo-second-order kinetic equations are used to analyze the adsorption kinetics. In this study, MFX and carbamazepine were reactants in the adsorption process. Based on the characteristics of MFX and the applicable scope of each kinetic equation, the pseudo-firstorder and pseudo-second-order kinetic equations were selected to fit the experimental data in this study.

In the first-order kinetics, the reaction rate is only proportional to the concentration of one reactant, while in the pseudo-first-order kinetics, the reaction rate is related to the concentration of the adsorbent and the adsorbate. When the concentration of one substance is much more than the

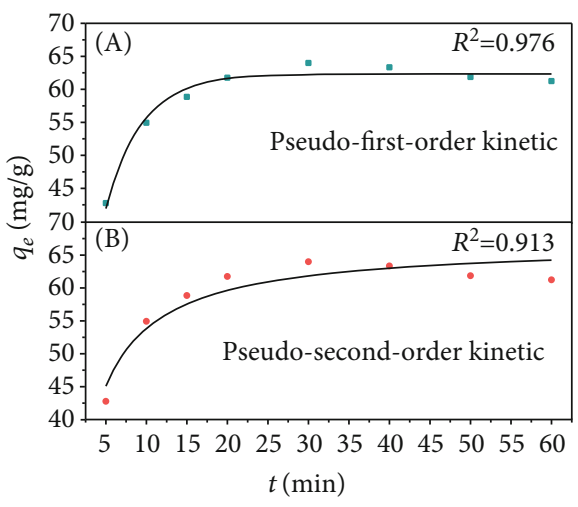

Figure 3: The fitting curve of the (a) pseudo-first-order kinetics and (b) pseudo-second-order kinetics.

other, it shows the characteristics of a first-order kinetic reaction $[29,30]$.

Figures 3(a) and 3(b) show the fitting results of the pseudo-first-order and pseudo-second-order kinetic equations. Table 1 shows the parameters of these kinetic equations. In Figure 3(a), the adsorption reaction reached adsorption equilibrium in $20 \mathrm{~min}$, and the maximum adsorption capacity was $62.32 \mathrm{mg}$ of carbamazepine per gram of MFX. In the actual experiment, the maximum adsorption capacity was $62.99 \mathrm{mg} / \mathrm{g}$ and the adsorption time was $30 \mathrm{~min}$, which were consistent with the values predicted by the pseudo-first-order kinetic equation. In Figure 3(b), the 
TABLE 1: The parameters of the pseudo-first-order and pseudo-second-order kinetics.

\begin{tabular}{|c|c|c|c|c|}
\hline Equation & Experimental $q_{e}(\mathrm{mg} / \mathrm{g})$ & Predicted $q_{e}(\mathrm{mg} / \mathrm{g})$ & $k_{1}$ & $R^{2}$ \\
\hline Pseudo-first-order kinetic & 62.99 & 62.32 & -0.22 & 0.98 \\
\hline Pseudo-second-order kinetic & 62.99 & 66.89 & 0.0062 & 0.91 \\
\hline
\end{tabular}

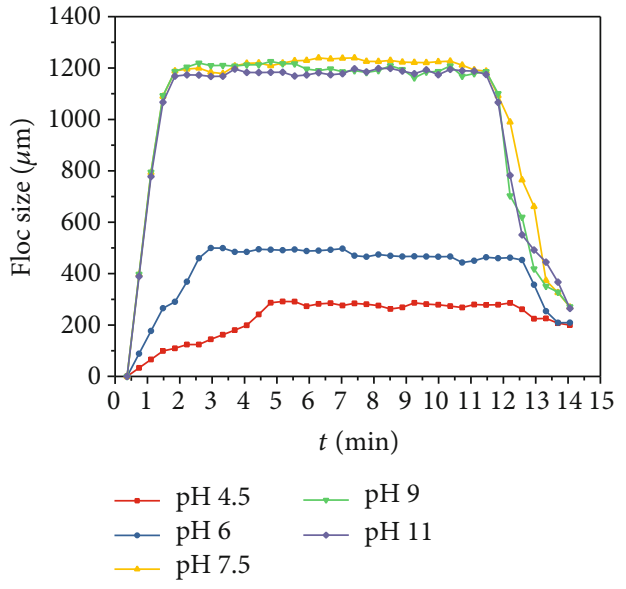

(a)

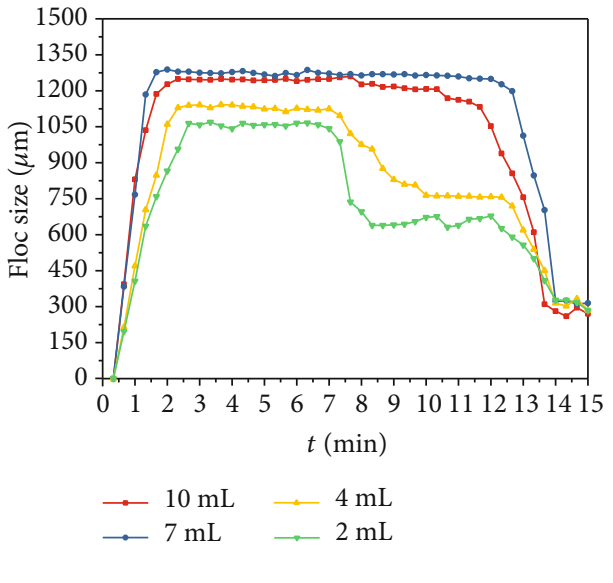

(b)

FIgUre 4: The effect of factors on floc formation: (a) $\mathrm{pH}$ and (b) dosage of MFX.

predicted adsorption did not reach adsorption equilibrium in $60 \mathrm{~min}$, and the predicted maximum adsorption capacity $(66.89 \mathrm{mg} / \mathrm{g}, 60 \mathrm{~min})$ was more than the actual value. Moreover, the $R^{2}$ of the pseudo-first-order kinetic equation is 0.976 , which is much higher than the $R^{2}$ of the pseudosecond-order kinetic equation. These results suggested that the adsorption of carbamazepine by MFX was more in line with the pseudo-first-order kinetic equation.

According to other studies, the adsorption process described by the first-order kinetic is controlled by substance transport. The pseudo-second-order kinetic simulates the second-order kinetic, which describes a chemical reaction, accompanied by electron sharing or transfer. The adsorption of carbamazepine by the MFX-fitted pseudo-first-order kinetic better than the pseudo-secondorder kinetic, which suggested that the adsorption was not pure chemical adsorption [17].

3.4. Mechanisms of Carbamazepine Adsorption on MFX. The preliminary experimental results showed that the $\mathrm{pH}$, dosage of MFX, dosage of coagulant, and temperature can affect the carbamazepine removal rate by MFX. The dosage of MFX and $\mathrm{pH}$ were the main factors; therefore, the flocculation morphology was used to further investigate the carbamazepine removal mechanism by MFX. The changes of floc size under different $\mathrm{pH}$ values/dosages of MFX at different times are shown in Figure 4. The flocs under different $\mathrm{pH}$ conditions showed a similar changing trend in the whole coagulation processes. The floc formation process was slow under acidic condition, and the particle size was relatively small in the stable stage. Moreover, there was no obvious sedimentation at $\mathrm{pH}=4.5$. The floc formation process was the fastest at
$\mathrm{pH}=7.5$. When the $\mathrm{pH}$ was larger than 7.5 , the growth of the flocs was significantly accelerated, the settling velocity of the flocs was fast, and the changes were not obvious as the $\mathrm{pH}$ became larger. This was because the surface charge density of MFX and carbamazepine was low under an alkalescent condition, which can weaken the repulsive force between carbamazepine and MFX and can help the growth and coherence of flocs [31].

In Figure 4(b), the maximum floc size was the smallest when the dosage of MFX was $2 \mathrm{~mL}(1064 \mu \mathrm{m})$, and the floc size became larger with the increase of MFX dosage. However, when the dosage was $10 \mathrm{~mL}(1235 \mu \mathrm{m})$, the floc size became smaller than that under a dosage of $7 \mathrm{~mL}$ $(1288 \mu \mathrm{m})$. Moreover, the growth of the floc was slower at a low dosage of MFX, and the maintenance time of the stable period was shorter, which was not conductive to the removal of carbamazepine. This was because too low of a dosage of MFX cannot provide sufficient adsorption sites for carbamazepine, and the collision probability between MFX and carbamazepine was low. Therefore, enough dosage of MFX can generate larger flocs. However, the dynamic equilibrium period was short with too high dosage of a MFX, because there was a large amount of the same charge with excess MFX, which increased electrostatic repulsion. Similar phenomena have been found in the study of the coagulation of microbial flocculant and kaolin, and this was caused by the adsorption bridging effect.

To investigate whether there was chemical reaction between carbamazepine and MFX, 3D-EEM and FTIR were used to analyze the samples before and after adsorption. Figures 5(a)-5(c) show the EEM of carbamazepine aqueous solution, precipitation after adsorption of carbamazepine by 


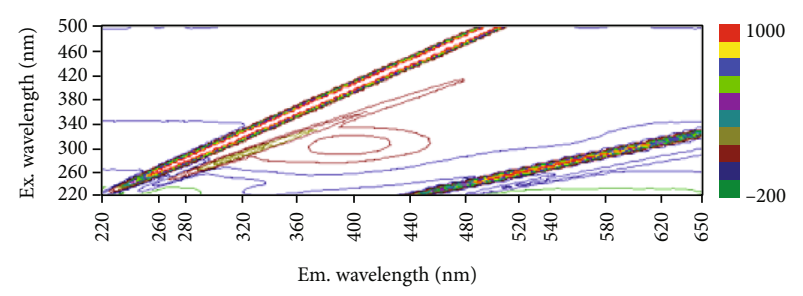

(a)

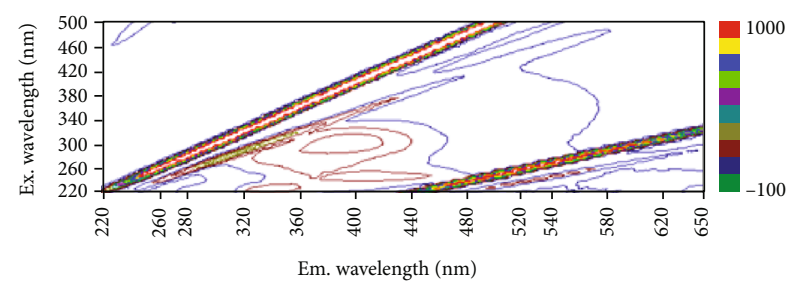

(c)

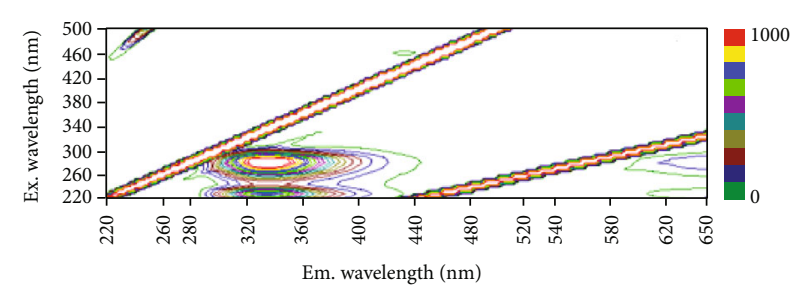

(b)

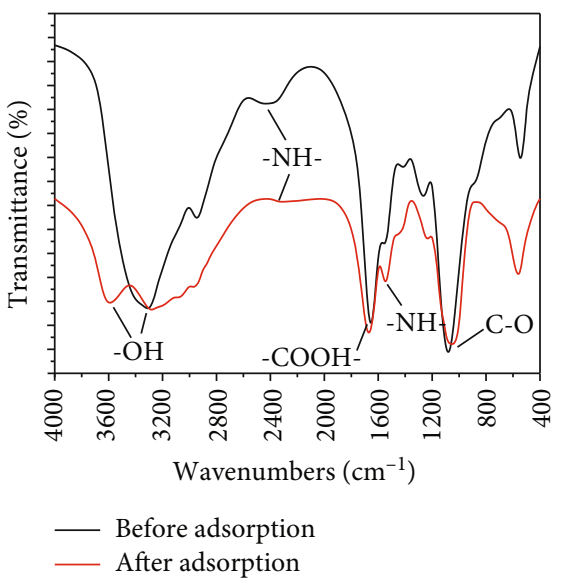

(d)

FIGURE 5: EEM of component of MFX adsorbing carbamazepine: (a) carbamazepine aqueous solution, (b) precipitation after adsorption, (c) liquid supernatant after adsorption, and (d) FTIR of MFX before and after carbamazepine.

MFX, and liquid supernatant after adsorption, respectively. In Figure 5(a), it can be seen that the carbamazepine aqueous solution only had an absorption peak at $\lambda \mathrm{ex} / \lambda \mathrm{em}=(310$ $320) \mathrm{nm} /(395-405) \mathrm{nm}$. This peak belongs to Class I (humic acid-like area), which was caused by typical exogenous organics. In this study, this peak was caused by carbamazepine [32]. In Figure 5(b), the precipitate after adsorption shows two high concentration absorption peaks at $\lambda \mathrm{ex} / \lambda \mathrm{em}$ $=(270-280) \mathrm{nm} /(325-335) \mathrm{nm}$ and $\lambda$ ex/ $/ \mathrm{em}=(225-235)$ $\mathrm{nm} /(325-335) \mathrm{nm}$, which are the characteristic peaks of proteins that are the main active components of MFX [20]. No characteristic peak of carbamazepine was found in this spectrum, because carbamazepine has been adsorbed on MFX through intermolecular forces and it was not a free molecule that can be detected. In Figure 5(c), three obvious absorption peaks can be seen in the spectrum of the supernatant after adsorption. The peak at $\lambda \mathrm{ex} / \lambda \mathrm{em}=(310-320) \mathrm{nm} /(395-405$ ) $\mathrm{nm}$ is the characteristic peak of carbamazepine. The peak at $\lambda$ ex $/ \lambda$ em $=(225-235) \mathrm{nm} /(325-335) \mathrm{nm}$ belongs to the tyrosine and benzene ring structure protein related to biological sources, which represent a small amount of the active ingredients of MFX or its derivatives. The peak related to tryptophan at $\lambda$ ex $/ \lambda \mathrm{em}=(250-280) \mathrm{nm} /(325-335) \mathrm{nm}$ disappeared, and a new peak resembling fulvic acid at $\lambda$ ex $/ \lambda$ $\mathrm{em}=(250-270) \mathrm{nm} /(410-420) \mathrm{nm}$ appeared. This peak was a new peak after MFX adsorbed carbamazepine, which suggested that chemical reactions occurred between carbamazepine and the tryptophan-like component on MFX.

To further prove that chemical reactions occurred between carbamazepine and MFX, FTIR was used to analyze MFX before and after adsorption of carbamazepine (Figure 5(d)).
Compared to the FTIR spectrum of MFX before adsorption, there was a new peak in the FTIR spectrum of MFX after adsorption, accompanied by multipeak displacement. The new peak appeared at $3590.22 \mathrm{~cm}^{-1}$, which was the characteristic peak corresponding to hydroxyl. The absorption peak caused by the stretching vibration of associative hydroxyl at $3309.94 \mathrm{~cm}^{-1}$ exhibited a redshift to $3291.88 \mathrm{~cm}^{-1}$, and the peak shape became wider. This proved that the hydroxyl played a role in the adsorption of carbamazepine by MFX, and this was achieved by hydrogen bonding. The absorption peak caused by the vibration of amino at $2435.06 \mathrm{~cm}^{-1}$ exhibited a redshift to $2321.08 \mathrm{~cm}^{-1}$, and the intensity was significantly weakened. The long and narrow absorption peak at $1654.45 \mathrm{~cm}^{-1}$ was a characteristic peak of carboxyl, and this peak exhibited a blueshift to $1669.71 \mathrm{~cm}^{-1}$. The peak at $1549.75 \mathrm{~cm}^{-1}$ caused by the bending vibration of amide exhibited a redshift to $1546.45 \mathrm{~cm}^{-1}$, which proved that the active amino in the proteins of MFX participated in the adsorption reaction. The long and narrow absorption peak at $1080.57 \mathrm{~cm}^{-1}$ as a typical absorption peak of sugar derivatives included the stretching and vibration of $\mathrm{C}-\mathrm{O}$ and $\mathrm{C}-\mathrm{O}-\mathrm{C}$. The peak exhibited a redshift to $1047.34 \mathrm{~cm}^{-1}$ after adsorption of carbamazepine. These results proved that the hydroxyl, amino, and carboxyl groups were all involved in the adsorption reaction and played a role together in the removal of carbamazepine by MFX [17].

\section{Conclusion}

MFX was used to remove carbamazepine. MFX was efficient in the removal of carbamazepine in both domestic sewage 
and secondary sedimentation tank effluent. The pseudo-firstorder kinetic equation can describe the adsorption process. The adsorption was a compound process that involved physical and chemical adsorption. MFX performed the best under alkalescent conditions, because the repulsive force between carbamazepine and MFX was weakened, which can help the growth and coherence of flocs. Enough dosage of MFX can generate larger flocs, but excessive dosage of MFX will decrease the carbamazepine removal efficiency due to the increase of electrostatic repulsion. The hydroxyl, amino, and carboxyl groups in MFX played an important role in the removal of carbamazepine. As a widely existing, nontoxic, and eco-friendly microbial flocculant, MFX has the potential for practical applications in carbamazepine removal from domestic sewage, carbamazepine wastewater, and natural water bodies.

\section{Data Availability}

The data that support the findings of this study are available from the corresponding author Jie Xing, upon reasonable request.

\section{Conflicts of Interest}

No potential conflict of interest exists in this work; therefore, nothing can affect the objectivity and actions of authors. The conflicts of interest, including direct financial relationships, indirect financial relationships, personal relationships, academic competition, and intellectual passion, are absolutely nonexistent in this work.

\section{Acknowledgments}

This work was financially supported by the National Science and Technology Major Project (2014ZX07201-012).

\section{References}

[1] L. Nielsen, P. Zhang, and T. J. Bandosz, "Adsorption of carbamazepine on sludge/fish waste derived adsorbents: effect of surface chemistry and texture," Chemical Engineering Journal, vol. 267, pp. 170-181, 2015.

[2] Y. Gao, G. Yu, K. Liu et al., "Integrated adsorption and visiblelight photodegradation of aqueous clofibric acid and carbamazepine by a Fe-based metal-organic framework," Chemical Engineering Journal, vol. 330, pp. 157-165, 2017.

[3] L. Nielsen, M. J. Biggs, W. Skinner, and T. J. Bandosz, "The effects of activated carbon surface features on the reactive adsorption of carbamazepine and sulfamethoxazole," Carbon, vol. 80, pp. 419-432, 2014.

[4] S. Jemutai-Kimosop, F. Orata, V. O. Shikuku, V. A. Okello, and Z. M. Getenga, "Insights on adsorption of carbamazepine onto iron oxide modified diatomaceous earth: kinetics, isotherms, thermodynamics, and mechanisms," Environmental Research, vol. 180, article 108898, 2020.

[5] Q. He, J. J. Liang, L. X. Chen et al., "Removal of the environmental pollutant carbamazepine using molecular imprinted adsorbents: molecular simulation, adsorption properties, and mechanisms," Water Research, vol. 168, article 115164, 2020.
[6] X. Gao, Q. Guo, G. Tang, W. Zhu, X. Yang, and Y. Luo, "TBAOH assisted synthesis of ultrathin BiOCl nanosheets with enhanced charge separation efficiency for superior photocatalytic activity in carbamazepine degradation," Journal of Colloid and Interface Science, vol. 570, pp. 242-250, 2020.

[7] J. Chen, D. Zhang, H. Zhang, S. Ghosh, and B. Pan, "Fast and slow adsorption of carbamazepine on biochar as affected by carbon structure and mineral composition," Sci Total Environ, vol. 579, pp. 598-605, 2017.

[8] K. Liu, L. Bai, Y. Shi et al., "Simultaneous disinfection of E. faecalis and degradation of carbamazepine by sulfate radicals: an experimental and modelling study," Environmental Pollution, vol. 263, article 114558, 2020.

[9] A. Li, C. Zhou, Z. Liu et al., "Direct solid-state evidence of $\mathrm{H}_{2}$ -induced partial U(VI) reduction concomitant with adsorption by extracellular polymeric substances (EPS)," Biotechnology and Bioengineering, vol. 115, no. 7, pp. 1685-1693, 2018.

[10] C. Y. Lai, Q. Y. Dong, J. X. Chen et al., "Role of extracellular polymeric substances in a methane based membrane biofilm reactor reducing vanadate," Environmental Science \& Technology, vol. 52, no. 18, pp. 10680-10688, 2018.

[11] J. Yang, D. Wu, A. Li et al., "The addition of N-hexanoylhomoserine lactone to improve the microbial flocculant production of Agrobacterium tumefaciens Strain $\mathrm{F}_{2}$, an exopolysaccharide bioflocculant-producing bacterium," Applied Biochemistry and Biotechnology, vol. 179, no. 5, pp. 728-739, 2016.

[12] D. Cui, C. Tan, H. Deng et al., "Biosorption mechanism of aqueous $\mathrm{Pb}^{2+}, \mathrm{Cd}^{2+}$, and $\mathrm{Ni}^{2+}$ Ions on extracellular polymeric substances (EPS)," Archaea, vol. 2020, Article ID 8891543, 9 pages, 2020.

[13] J. Yang, L. Zhou, F. Ma et al., "Magnetic nanocomposite microbial extracellular polymeric substances@ $\mathrm{Fe}_{3} \mathrm{O}_{4}$ supported $\mathrm{nZVI}$ for $\mathrm{Sb}(\mathrm{V})$ reduction and adsorption under aerobic and anaerobic conditions," Environmental Research, vol. 189, article 109950, 2020.

[14] W. Wei, Q. Wang, A. Li et al., "Biosorption of Pb (II) from aqueous solution by extracellular polymeric substances extracted from Klebsiella sp. J1: adsorption behavior and mechanism assessment," Scientific Reports, vol. 6, no. 1, article 31575, 2016.

[15] W. Wei, A. Li, J. Yang et al., "Synergetic effects and flocculation behavior of anionic polyacrylamide and extracellular polymeric substrates extracted from Klebsiella sp. J1 on improving soluble cadmium removal," Bioresource Technology, vol. 175, pp. 34-41, 2015.

[16] L. Zhou, A. Li, F. Ma, J. Yang, S. Pi, and A. Tang, "Sb(V) reduced to $\mathrm{Sb}(\mathrm{III})$ and more easily adsorbed in the form of $\mathrm{Sb}(\mathrm{OH}) 3$ by microbial extracellular polymeric substances and core-shell magnetic nanocomposites," ACS Sustainable Chemistry \& Engineering, vol. 7, no. 11, pp. 10075-10083, 2019.

[17] W. Wei, A. Li, S. Pi et al., "Synthesis of core-shell magnetic nanocomposite $\mathrm{Fe}_{3} \mathrm{O}_{4} @$ microbial extracellular polymeric substances for simultaneous redox sorption and recovery of silver ions as silver nanoparticles," ACS Sustainable Chemistry \& Engineering, vol. 6, no. 1, pp. 749-756, 2017.

[18] L. Zhou, A. Li, F. Ma et al., "Combining high electron transfer efficiency and oxidation resistance in nZVI with coatings of microbial extracellular polymeric substances to enhance $\mathrm{Sb}(\mathrm{V})$ reduction and adsorption," Chemical Engineering Journal, vol. 395, article 125168, 2020.

[19] J. Xing, J. X. Yang, A. Li et al., "Removal efficiency and mechanism of sulfamethoxazole in aqueous solution by 
bioflocculant MFX," Journal of Analytical Methods in Chemistry, vol. 2013, Article ID 568614, 8 pages, 2013.

[20] S. Pi, A. Li, D. Cui et al., "Biosorption behavior and mechanism of sulfonamide antibiotics in aqueous solution on extracellular polymeric substances extracted from Klebsiella sp. J1," Bioresource Technology, vol. 272, pp. 346-350, 2019.

[21] S. Pi, A. Li, W. Wei et al., "Synthesis of a novel magnetic nanoscale biosorbent using extracellular polymeric substances from Klebsiella sp. J1 for tetracycline adsorption," Bioresource Technology, vol. 245, pp. 471-476, 2017.

[22] J. Yang, W. Wei, S. Pi et al., "Competitive adsorption of heavy metals by extracellular polymeric substances extracted from Klebsiella sp. J1," Bioresource Technology, vol. 196, pp. 533539, 2015.

[23] M. Yao, L. Duan, J. Wei, F. Qian, and S. W. Hermanowicz, "Carbamazepine removal from wastewater and the degradation mechanism in a submerged forward osmotic membrane bioreactor," Bioresource Technology, vol. 314, article 123732, 2020.

[24] J. Huang, Z.-L. Huang, J.-X. Zhou et al., "Enhancement of heavy metals removal by microbial flocculant produced by Paenibacillus polymyxa combined with an insufficient hydroxide precipitation," Chemical Engineering Journal, vol. 374, pp. 880-894, 2019.

[25] D.-Q. Cao, W.-Y. Yang, Z. Wang, and X. D. Hao, "Role of extracellular polymeric substance in adsorption of quinolone antibiotics by microbial cells in excess sludge," Chemical Engineering Journal, vol. 370, pp. 684-694, 2019.

[26] W. Wei, A. Li, F. Ma et al., "Simultaneous sorption and reduction of $\mathrm{Cr}(\mathrm{VI})$ in aquatic system by microbial extracellular polymeric substances fromKlebsiellasp. J1," Journal of Chemical Technology \& Biotechnology, vol. 93, no. 11, pp. 3152-3159, 2018.

[27] Z. Yang, M. Li, M. Yu et al., "A novel approach for methylene blue removal by calcium dodecyl sulfate enhanced precipitation and microbial flocculant GA1 flocculation," Chemical Engineering Journal, vol. 303, pp. 1-13, 2016.

[28] P. Sivaperumal, K. Kamala, and R. Rajaram, “Adsorption of cesium ion by marine actinobacterium Nocardiopsis sp. $13 \mathrm{H}$ and their extracellular polymeric substances (EPS) role in bioremediation," Environmental Science and Pollution Research International, vol. 25, no. 5, pp. 4254-4267, 2018.

[29] A. A. Marianou, C. M. Michailof, D. K. Ipsakis et al., "Isomerization of glucose into fructose over natural and synthetic $\mathrm{MgO}$ catalysts," ACS Sustainable Chemistry \& Engineering, vol. 6 , no. 12, pp. 16459-16470, 2018.

[30] Y. Zhou, Y.S. Lai, E. Eustance, and B. E. Rittmann, "Promoting Synechocystis sp. PCC 6803 harvesting by cationic surfactants: alkyl-chain length and dose control for the release of extracellular polymeric substances and biomass aggregation," ACS Sustainable Chemistry \& Engineering, vol. 7, no. 2, pp. 21272133, 2018.

[31] Y. X. Zhao, B. Y. Gao, H. K. Shon, J. H. Kim, Q. Y. Yue, and Y. Wang, "Floc characteristics of titanium tetrachloride $\left(\mathrm{TiCl}_{4}\right)$ compared with aluminum and iron salts in humic acid-kaolin synthetic water treatment," Separation and Purification Technology, vol. 81, no. 3, pp. 332-338, 2011.

[32] L. Liang, S. Gao, J. Zhu et al., "The enhanced photocatalytic performance toward carbamazepine by nitrogen-doped carbon dots decorated on $\mathrm{BiOBr} / \mathrm{CeO}_{2}$ : mechanism insight and degradation pathways," Chemical Engineering Journal, vol. 391, article 123599, 2020. 